\title{
Crossed Monopole Array for Single Mode Excitation of Ventilation Duct Communication Channels
}

\author{
Benjamin E. Henty ${ }^{1}$, Jessica Hess ${ }^{2}$, and Daniel D. Stancil ${ }^{2}$ \\ ${ }^{1}$ i5wireless, LLC., Lanham, MD, USA, E-mail: henty+aps@eirp.org \\ ${ }^{2}$ Carnegie Mellon University, Pittsburgh, PA, USA, \\ E-mail: jessica.hess@gmail.com, stancil@cmu.edu
}

\begin{abstract}
This work presents a novel antenna array for exciting a single mode of propagation in common ventilation ducts with a circular cross-section that are overmoded waveguides in the $2.4 \mathrm{GHz}$ frequency band. By inverting the phase of one driven element with respect to the other, one of two modes can be selected for excitation, but in either case only a single mode is excited. We present the design technique and advantages of such an antenna array for use with IEEE 802.11g Access Points.
\end{abstract}

\section{Introduction}

Ventilation ducts provide a unique means for distributing IEEE $802.11 \mathrm{~b} / \mathrm{g}$ signals indoors. Typical sized ventilation ducts used at $2.4 \mathrm{GHz}$ act as overmoded waveguides. While this results in low loss, it can also lead to long delay spreads, as each mode excited by an antenna has a different propagation velocity in the duct. IEEE 802.11g networks adjust the data rate based on the channel quality, which is degraded when there is a long delay spread.

One way to reduce the delay spread and possibly increase the throughput over an IEEE $802.11 \mathrm{~g}$ link through ventilation ducts is to use an antenna that excites only one mode of propagation. Towards this end, a new antenna array designed to excite primarily a single mode has been developed. The array is practical for easy installation in ventilation ducts and can be incorporated to complement existing two antenna diversity techniques in current IEEE 802.11 Access Points (APs). The array is practical for easy installation in ventilation ducts and can be incorporated to complement existing 2 antenna diversity techniques in current IEEE 802.11 Access Points (AP).

\section{Single Mode Antenna Array}

The antennas are designed to operate in the $2.4 \mathrm{GHz}$, ISM (Industrial, Scientific and Medical) frequency band using $315 \mathrm{~mm}$ diameter ducts. The $2.4 \mathrm{GHz}$ frequency band is allocated throughout the world, while we have encountered the $315 \mathrm{~mm}$ diameter frequently in our installations in Scandinavia. A similar $305 \mathrm{~mm}$ diameter is available in the United States. For the $2.4 \mathrm{GHz}$ frequency band, 18 modes can propagate in the $315 \mathrm{~mm}$ diameter duct. Most unfortunately, the cutoff frequency of the highest order mode in the $315 \mathrm{~mm}$ diameter duct falls in the $2.4 \mathrm{GHz}$ frequency band, potentially causing sharp nulls in the frequency range of interest. 
The antenna array design uses two identical, properly driven array elements to excite a single mode. Thus, we begin by analyzing the mode excitation of a single array element and then analyze how the elements are driven to excite primarily a single mode. In [1], Nikitin used a technique to vary the element length of a monopole antenna connected to a cylindrical duct and search for a length that would excite primarily a single mode. In this work we have instead searched for a length of an antenna which excites primarily two modes. For a $315 \mathrm{~mm}$ diameter duct, an antenna length of interest is $205 \mathrm{~mm}$. Using simulation software developed by Nikitin in [1] and based on the theory presented in [2], we have computed the expected mode content excited by such a monopole of this length.

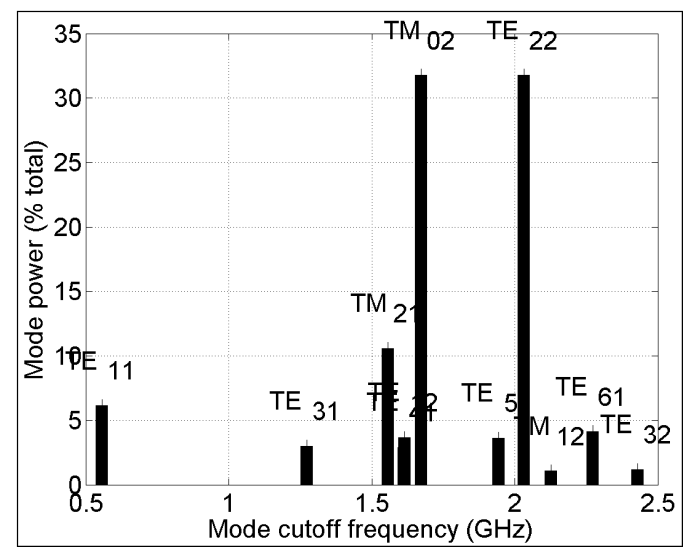

Figure 1: Modes excited by our long monopole antenna element according to software written by Nikitin [1].

As seen in Figure 1, with a monopole length of $205 \mathrm{~mm}$, the $T E_{22}$ and $T M_{02}$ modes are present equally. Further, the potentially problematic $T E_{32}$ mode, with a cutoff frequency in the ISM band, is only slightly present. With this advantage, we next consider the field patterns of the two major modes present in the setup, which are shown in Figure 2 as excerpted from [3].

The electric field (E-field) patterns of the two modes shown in Figure 2 present an intriguing opportunity. Since the E-field pattern of the $T M_{02}$ mode is circularly symmetric, but the E-field pattern of the $T E_{22}$ mode alternates in sign in each quadrant of the cross-section, a circumferential antenna array with elements spaced 90 degrees apart around the diameter is useful for providing the desired mode selectivity. In this geometry, each array element can be thought of as exciting both the $T M_{02}$ and $T E_{22}$ modes with relative phases determined by the field patterns of the modes. For example, if two $205 \mathrm{~mm}$ long monopoles were driven in phase, both $T M_{02}$ modes would be excited in phase resulting in a strong mode excitation. However, the radial component of the $T E_{22}$ mode reverses sign every 90 degrees around the circumference so the modes excited by the array elements will be 180 degrees out of phase and cancel. Various other modes will be enhanced or degraded, but the result should be a mode mixture dominated by the $T M_{02}$ mode. Likewise, if the array elements are driven out of phase by 180 degrees, the mode mix will be dominated by $T E_{22}$. 


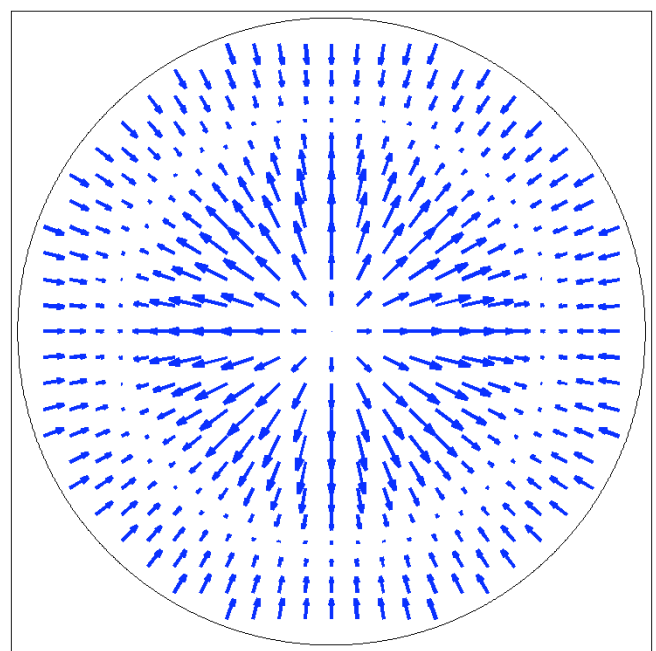

(a) $T M_{02}$ Electric field

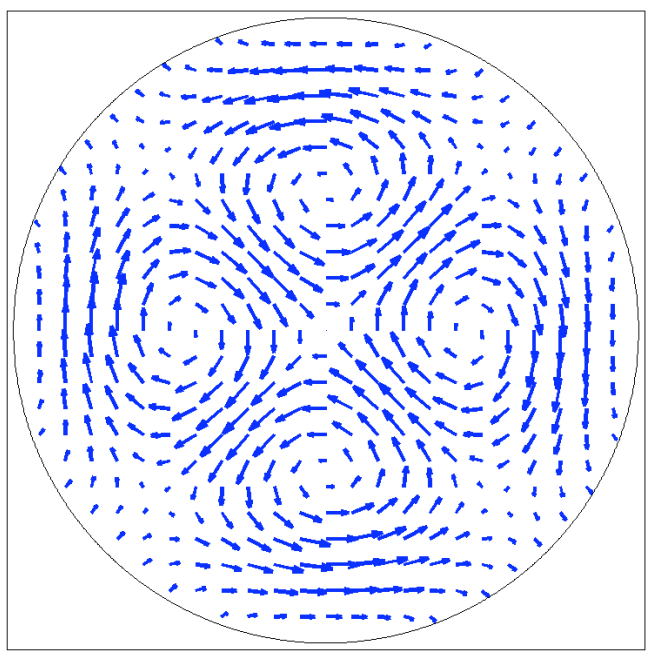

(b) $T E_{22}$ Electric field

Figure 2: E-field patterns of the major modes excited by a monopole $205 \mathrm{~mm}$ long in a $315 \mathrm{~mm}$ diameter cylindrical duct. Arrows indicate the magnitude and direction of the field in the plane of the cross-section. Inspired by [4].

This idea for an antenna array is quite compelling. Since both elements need only be driven either 0 or 180 degrees out of phase, the array can be implemented with minimal cost. In addition, monopole antennas can be easily installed in a ventilation duct. The phase shifts of 0 and 180 degrees can of course be obtained by simply ensuring the antenna connections are made with equal or opposite polarities. However, one could also use a 180 degree hybrid power splitter for an even greater advantage as shown in Figure 3. If you connect the AP antenna ports to the sum and difference ports of the hybrid, then each AP antenna port will excite a single mode in the duct, but each port will excite a different single mode. This is because the signal on the sum port will excite the duct antennas in phase and excite the $T M_{02}$ mode and the difference port will excite the duct antennas 180 degrees out of phase and excite the $T E_{22}$ mode.

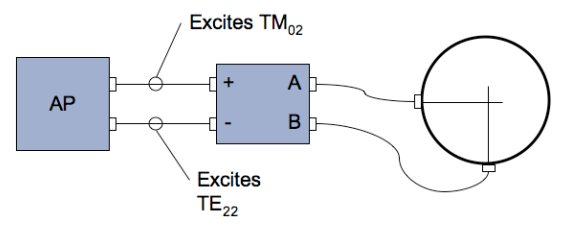

Figure 3: Diagram of how to use a 180 degree hybrid splitter to excite different single modes depending on which AP antenna port is used.

The major downside of this antenna is that it is not matched to a $50 \mathrm{ohm}$ impedance system. Thus, in order to use the antenna, the user must be willing to contend with a higher insertion loss in the system or impedance matching circuitry needs to be provided. There is a potential, though, to combine impedance matching circuitry with a 180 degree hybrid power splitter to maximize the efficiency and minimize the hardware installed with the antennas. 
To validate the concept of the antenna array for exciting single modes of propagation, we performed measurements on an experimental long monopole array. The measurements were conducted in a straight run of cylindrical, $0.3048 \mathrm{~m}$ diameter duct. The duct used was $3 \mathrm{~m}$ long and the ends were left open. The antenna on one end was always a quarter-wavelength monopole. The other antenna was either another quarter-wavelength monopole or a 2 element, long monopole array with the elements driven in phase. The duct channel response for these two different antennas was recorded using a vector network analyzer at 1601 points between 2.4 and $2.5 \mathrm{GHz}$. The responses are plotted in Figure 4.
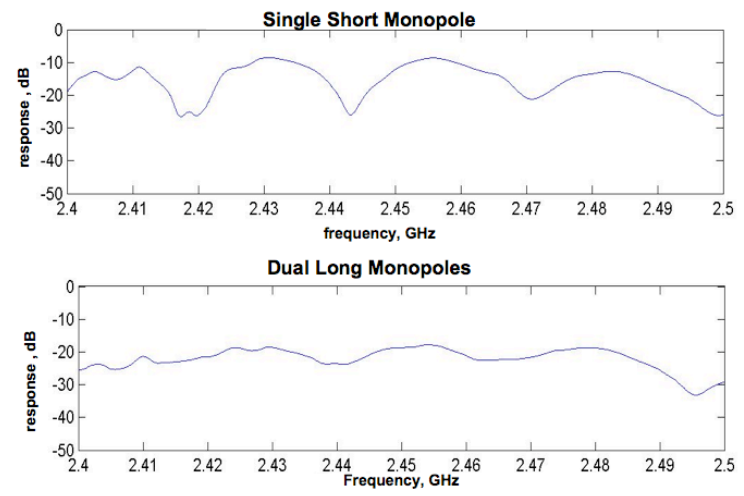

Figure 4: Measured frequency responses comparing a quarter-wavelength monopole in the top plot and a long monopole array in the bottom plot.

As seen in Figure 4, the peak-to-peak variation of the channel has been significantly reduced through the use of the long monopole array. For the ISM band, between 2.4 and $2.4835 \mathrm{GHz}$, the peak-to-peak variation in the frequency response is reduced from $18 \mathrm{~dB}$ for the short monopole to $8 \mathrm{~dB}$ for the long monopole antenna array. While this does not directly tell us the mode content present, it does indicate that the response is dominated by a single propagating mode and that the array is functioning as expected.

\section{References}

[1] P. V. Nikitin, "Analysis of heating, ventilation and air conditioning ducts as a radio frequency communication channel," Ph.D. dissertation, Carnegie Mellon University, Pittsburgh, PA, Aug. 2002.

[2] P. Nikitin, D. Stancil, A. Cepni, A. Xhafa, O. Tonguz, and D. Brodtkorb, "A novel mode content analysis technique for antennas in multimode waveguides," IEEE Transactions on Microwave Theory and Techniques, vol. 51, no. 12, pp. 2402- 2408, Dec 2003.

[3] C. A. Balanis, Advanced Engineering Electromagnetics. John Wiley \& Sons, 1989.

[4] C. S. Lee, S. W. Lee, and S. L. Chuang, "Plot of modal field distribution in rectangular and circular waveguides," IEEE Transactions on Microwave Theory and Techniques, vol. 33, no. 3, pp. 271-274, Mar. 1985. 